Relations industrielles

Industrial Relations

\title{
Le coût de la Sécurité Sociale, 1949-1954, Bureau International du Travail, Genève, 1958, 204 pp. \$3.00.
}

\section{Émile Gosselin}

Volume 14, numéro 4, octobre 1959

URI : https://id.erudit.org/iderudit/1022138ar

DOI : https://doi.org/10.7202/1022138ar

Aller au sommaire du numéro

Éditeur(s)

Département des relations industrielles de l'Université Laval

ISSN

0034-379X (imprimé)

1703-8138 (numérique)

Découvrir la revue

Citer ce compte rendu

Gosselin, É. (1959). Compte rendu de [Le coût de la Sécurité Sociale, 1949-1954,

Bureau International du Travail, Genève, 1958, 204 pp. \$3.00.] Relations

industrielles / Industrial Relations, 14(4), 619-620.

https://doi.org/10.7202/1022138ar

Tous droits réservés (C Département des relations industrielles de l’Université Laval, 1959
Ce document est protégé par la loi sur le droit d'auteur. L’utilisation des services d'Érudit (y compris la reproduction) est assujettie à sa politique d'utilisation que vous pouvez consulter en ligne.

https://apropos.erudit.org/fr/usagers/politique-dutilisation/ 
syndicats. Les rapports sont beaucoup plus amicaux en Europe. \&What is, perhaps, most paradoxical is that industrial relations in countries with a far less egalitarian social structure than America, and in which trade unions have a strong ideological character, should be less marked by bitter antagonism. » Il s'étend longuement sur la négociation, l'arbitrage et la grève et constate que de part et d'autre on est devenu prisonnier d'un comportement qui s'est institutionnalisé. Bien que la négociation collective sur le plan de l'usine soit beaucoup plus poussée qu'en Europe, «American management appears to be far more determined to maintain its past prerogatives than management in Europe ». On se bat encore pour des pseudo-principes alors que l'on devrait travailler à trouver un modus vivendi avantageux pour tous. La démocratisation de l'industrie est encore à venir. Si la dictature industrielle a été peu à peu modifiée dans le sens de la démocratie par l'avènement des unions, les principes fondamentaux qui inspirent la direction des entreprises sont encore de type totalitaire. Ce n'est certainement pas avec un tel système que les Etats-Unis réussiront à attirer dans leur orbitre les nations d'Afrique et d'Asie qui répugnent à accepter cet aspect autoritaire du capitalisme privé.

Enfin l'auteur compare l'action politique des syndicats américains avec celle des syndicats anglais. Il se montre franchement favorable à une action politique qui sauvegarde l'autonomie de chacune de ces institutions. Il est très dangereux d'empêcher les syndicats de faire de la politique. \& The result of any such action would almost certainly be to stimulate the unions to much greater industrial militancy. What the unions would be deprived of seeking through democratic political action, they would be compelled to struggle for by other means. The possible repercussions ought to be pondered carefully before any attempt to prohibit the political activities of the unions is seriously contemplated. \$ Quand on critique les unions parce qu'elles flirtent du coté politique, on doit se souvenir qu'elles ont eu à lutter durement pour conquérir tout ce qu'elles possèdent de droits: droit d'exister, droit de négocier, droit de grève, droit de participer à l'élaboration de la législation qui affecte vitalement les intérêts économiques et sociaux de leurs membres. Dans tous les pays, les unions jouent un rôle important dans la politique. Alors il ne faudna pas se surprendre si, de plus en plus, les syndicats américains auront à abandonner leur traditionnelle attitude de neutralité, sans pourtant devenir prisonniers d'un parti.

Ainsi qu'on aura pu le constater, l'auteur a abordé tous les aspects du syndicalisme américain qui font à l'heure actuelle objet de discussions acerbes. Aussi nous souhaitons que son ouvrage soit hu par tous ceux qui se mêlent de parler du syndicalisme souvent à tort et à travers. On peut ne pas partager toutes les opinions de l'auteur, mais celles-ci valent la peine d'être soigneusement considérées.

Gérard Dion

Le coût de la Sécurité Sociale, 19491954, Bureau International du Travail, Genève, 1958, 204 pp. \$3.00.

Depuis deux décades, des systèmes de sécurité sociale sont apparus très nombreux dans la plupart des pays. Ils affectent considérablement l'économie de ces pays et il est très utile de connaître à fond les problèmes financiers des divers systèmes afin de calculer leurs effets sur l'économie nationale de chaque nation.

Le Bureau International du Travail a accompli un travail considérable et dont le besoin était urgent dans le domaine de la compilation, l'analyse et la diffusion des données sur le coût de la sécurité sociale à travers le monde. En 1952 et en 1955 le B.I.T. publiait ses deux premières études à ce sujet.

L'étude publiée en 1958 répondait à deux préoccupations. \& Il s'agissait en premier lieu », souligne l'introduction, «de présenter, par pays, un état complet et coordonné des opérations financières des régimes de sécurité sociale considérée comme rentrant dans le champ de l'enquête. En deuxième lieu l'étude est une étape en vue d'établir une comparaison internationale de la répartition du coût de la sécurité sociale. 
Le lecteur trouvera dans l'introduction à quels critères doit répondre un système pour pouvoir être considéré comme faisant partie du système national de sécurité sociale. Dans une première partie, l'étude présente, pour 32 pays, les tableaux de base indiquant les divers postes de recettes et de dépenses.

Dans une deuxième partie on essaie d'établir, à l'aide de tableaux, la comparaison internationale des données de la sécurité sociale. La troisième partie comporte une série très précieuse de tableaux sur diverses formes de sécurité sociale propres à divers pays.

Ce recueil doit faire partie de la bibliothèque de tous ceux que leur activité économique ou leur situation sociale obligent à s'occuper de l'élaboration ou de la mise en application de systèmes de sécurité sociale publics ou privés. Economistes, sociologues, spécialistes du travail y trouveront également une source inestimable de renseignements rigoureusement controlés et à date.

EMme Gosseln

Le cô̂t des soins médicaux, Bureau International du Travail, Genève, 1959, $260 \mathrm{pp}$.

Dans l'étude intitulée Le coût des soins médicaux », travail présenté avec extrêmement de minutie, le Bureau International du Travail a réuni une forte documentation relative aux différents soins médicaux et à leur coût total dans quatorze pays qui possiecient des services de soins médicaux étendus. Une attention spéciale est portée à la situation aux Etats-Unis. Les statistiques à ce sujet ont été ondonnés de façon à permettre au lecteur de comparer les coûts non seulement à l'intérieur d'un même pays mais d'un pays à l'autre.

Le volume comporte cinq chapitres et plusieurs annexes où l'on donne le détail des calculs par pays, la répartition des dépenses publiques et privées et l'affectation de ces dépenses selon les services rendus, ainsi qu'une série de tableaux comparatifs indiquant le nombre de personnes protégées pour chacun des régimes de sécurité sociale et les dépenses pour prestations médicales par personne protégée.

Dans le ler chapitre de l'ouvrage, l'on entend préciser l'étendue de la protection médicale en couvrant deux aspects: le nombre et les catégories de personnes protégées selon chaque pays, les risques ou éventualités couverts par les différents systèmes, et les répercussions que peuvent représenter ces deux facteurs si on les évalue différemment d'un pays à l'autre sur la comparabilité des dépenses pour prestations médicales. Nous recommandons très particulièrement $\propto$ chapître à ceux qui ont à établir ou à négocier des plans à l'intention de catégories de personnes bien définies.

Dans le deuxième chapître on évalue pour chaque pays la dépense pour diverses prestations médicales ainsi que le coût des soins médicaux reçus par les personnes protégées par opposition aux dépenses des institutions de sécurité sociale pour ces mêmes soins.

Dans le troisième chapître, l'on tente de dégager pour une période donné, l'évolution des dépenses pour prestations médicales selon les différents régimes de sécurité sociale offrant une protection médicale quelconque et de situer chaque catégorie de soins par rapport aux autres au point de vue coût.

Le chapitre IV analyse le revenu que les médecins, et dentistes tirent de la sécurité sociale et d'autres sources. Les conclusions de ce chapître en regard des chapîtres précédents s'avéreraient très utiles, en particulier pour les employeurs et les associations professionnelles qui ont à négocier en vue d'établir les barêmes de rémunération pour les personnes affectées aux soins médicaux protégés par les régimes de sécurité sociale.

Enfin le chapitre V tire les conclusions de l'analyse faite au cours des chapitres précédents. On notera en particulier dans quelle mesure le coût des divers soins médicaux affecte le revenu moyen, à quoi et dans quelle proportion sont affectées les diverses dépenses: hospitalisation, médecins, spécialistes, remèdes, dentistes. Quant à la tendance des dépenses, il faut souligner que sauf en France et en 